\title{
Mutations of SARS-CoV-2 nsp14 exhibit strong association with increased genome-wide mutation load
}

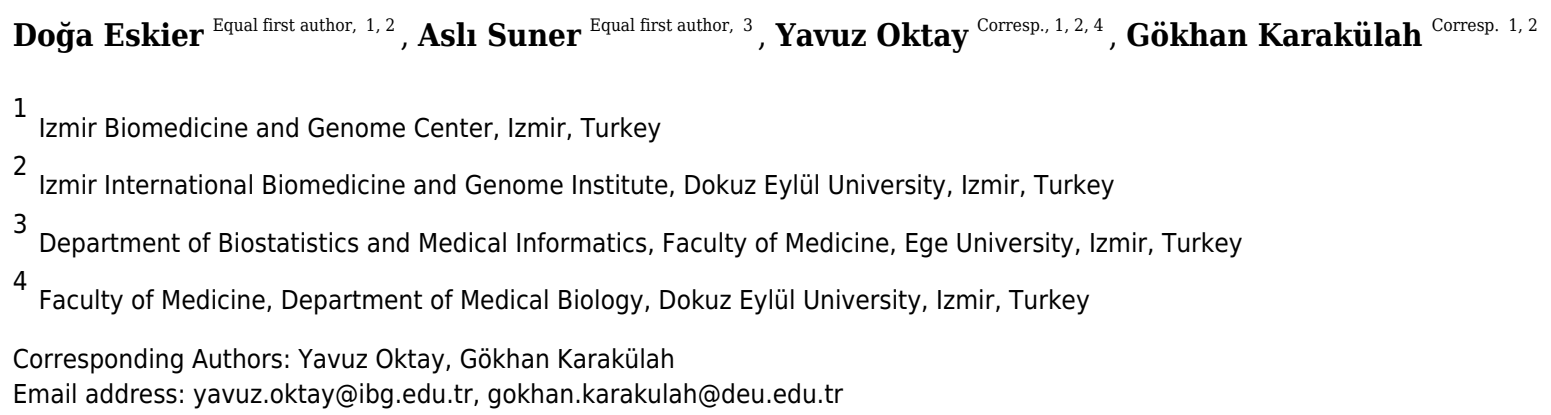

SARS-CoV-2 is a betacoronavirus responsible for human cases of COVID-19, a pandemic with global impact that first emerged in late 2019. Since then, the viral genome has shown considerable variance as the disease spread across the world, in part due to the zoonotic origins of the virus and the human host adaptation process. As a virus with an RNA genome that codes for its own genomic replication proteins, mutations in these proteins can significantly impact the variance rate of the genome, affecting both the survival and infection rate of the virus, and attempts at combating the disease. In this study, we analyzed the mutation densities of viral isolates carrying frequently observed mutations for four proteins in the RNA synthesis complex over time in comparison to wildtype isolates. Our observations suggest mutations in nsp14, an error-correcting exonuclease protein, have the strongest association with increased mutation load in both regions without selective pressure and across the genome, compared to nsp7, 8, and 12, which form the core polymerase complex. We propose nsp14 as a priority research target for understanding genomic variance rate in SARS-CoV-2 isolates, and nsp14 mutations as potential predictors for high mutability strains. 
1 Mutations of SARS-CoV-2 nsp14 exhibit strong association with increased genome-wide

2 mutation load

3

4 Doğa Eskier ${ }^{1,2, *}$, Aslı Suner ${ }^{3, *}$, Yavuz Oktay ${ }^{1,2,4, \dagger}$, Gökhan Karakülah ${ }^{1,2, \dagger}$

5

$6 \quad{ }^{1}$ Izmir Biomedicine and Genome Center (IBG), Izmir, Turkey

7 2Izmir International Biomedicine and Genome Institute, Dokuz Eylül University, Izmir, Turkey

$8{ }^{3}$ Department of Biostatistics and Medical Informatics, Faculty of Medicine, Ege University,

9 Izmir, Turkey

$10{ }^{4}$ Faculty of Medicine, Department of Medical Biology, Dokuz Eylül University, Izmir, Turkey

11 *These authors contributed equally to the work presented here and should therefore be regarded as

12 first authors.

13

$14 \dagger$ Co-corresponding authors:

15 Gökhan Karakülah, E-mail: gokhan.karakulah@deu.edu.tr

16 Yavuz Oktay, E-mail: yavuz.oktay@ibg.edu.tr

17 


\section{Abstract}

19 SARS-CoV-2 is a betacoronavirus responsible for human cases of COVID-19, a pandemic with 20 global impact that first emerged in late 2019. Since then, the viral genome has shown

21 considerable variance as the disease spread across the world, in part due to the zoonotic origins

22 of the virus and the human host adaptation process. As a virus with an RNA genome that codes

23 for its own genomic replication proteins, mutations in these proteins can significantly impact the

24 variance rate of the genome, affecting both the survival and infection rate of the virus, and

25 attempts at combating the disease. In this study, we analyzed the mutation densities of viral

26 isolates carrying frequently observed mutations for four proteins in the RNA synthesis complex

27 over time in comparison to wildtype isolates. Our observations suggest mutations in nsp14, an

28 error-correcting exonuclease protein, have the strongest association with increased mutation load

29 in both regions without selective pressure and across the genome, compared to nsp 7,8 , and 12 ,

30 which form the core polymerase complex. We propose nsp14 as a priority research target for

31 understanding genomic variance rate in SARS-CoV-2 isolates, and nsp14 mutations as potential

32 predictors for high mutability strains.

33 Keywords: SARS-CoV-2, COVID-19, nsp14, 3'-5' exonuclease, RNA-dependent RNA

34 polymerase, RdRp, mutation rate

\section{Introduction}

36 COVID-19 is an ongoing global pandemic characterized by long-term respiratory system

37 damage in patients, and caused by the SARS-CoV-2 betacoronavirus. It is likely of zoonotic

38 origin, but capable of human-to-human transmission, and since the first observed cases in the

39 Wuhan province of China (Chan et al., 2020; Riou \& Althaus, 2020), it has infected over 14

40 million people, with 612,054 recorded deaths (as of 22 July 2020). In addition to its immediate 
41 effects on the respiratory system, its long term effects are still being researched, including

42 symptoms such as neuroinvasion ( $\mathrm{Li}$, Bai \& Hashikawa, 2020; Wu et al., 2020), cardiovascular complications (Kochi et al., 2020; Zhu et al., 2020), and gastrointestinal and liver damage (Lee, Huo \& Huang, 2020; Xu et al., 2020). Due to its high transmissibility, and capacity for asymptomatic transmission (Wong et al., 2020), study of COVID-19 and its underlying pathogen remain a high priority. As a result, the high amount of frequently updated data on viral genomes on databases such as GISAID (Elbe \& Buckland-Merrett, 2017) and NextStrain (Hadfield et al., 2018) provides researchers with invaluable resources to track the evolution of the virus as it spreads across the world.

SARS-CoV-2 has a linear, single-stranded RNA genome, and does not depend on host proteins for genomic replication, instead using an RNA synthesis complex formed from nonstructural proteins (nsp) coded by its own genome. Four of the key proteins involved in the complex are nsp7, nsp8, nsp12, and nsp14, all of which are formed from cleavage of the polyprotein Orf1ab

54 into mature peptides. Nsp12, also known as RdRp (RNA-dependent RNA polymerase), is responsible for synthesizing new strands of RNA using the viral genome as a template. Nsp7 and nsp8 act as essential co-factors for the polymerase unit, together creating the core polymerase complex (Kirchdoerfer \& Ward, 2019; Peng et al., 2020), while nsp14 is an exonuclease which provides error-correcting capability to the RNA synthesis complex, therefore allowing the SARS-CoV-2 to maintain its large size genome (Subissi et al., 2014; Ma et al., 2015; Ogando et al., 2019; Romano et al., 2020). Owing to their role in maintaining replication fidelity and

61 directly affecting the mutation-selection equilibrium of RNA viruses, these proteins are key 62 targets of study in understanding the mutation accumulation and adaptive evolution of the virus 63 (Eckerle et al., 2010; Peng et al., 2020). 
64 In our previous study, we examined the top 10 most frequent mutations in the SARS-CoV-2

65 nsp12, and identified that four of them are associated with an increase in mutation density in two

66 genes, the membrane glycoprotein $(\mathrm{M})$ and the envelope glycoprotein (E) (the combination of

67 which is hereafter referred to as MoE, as we previously described), which are not under selective

68 pressure, and mutations in these genes are potential markers of reduced replication fidelity

69 (Eskier et al., 2020a). In this study, we follow up on our previous findings and analyze the

70 mutations in nsps 7,8 , and 14 , in addition to nsp12, to identify whether the mutations are

associated with a nonselective increase in mutation load or not. We then examine whole genome

mutation densities in mutant isolates in comparison to wildtype isolates using linear regression

models, in order to understand whether the mutations are associated with potential functional

impact. Our findings indicate that mutations in nsp14 are most likely to be predictors of

accelerated mutation load increase.

76

77

\section{Materials and Methods}

\section{Genome sequence filtering, retrieval, and preprocessing}

As previously described (Eskier et al., 2020a), SARS-CoV-2 isolate genome sequences and the corresponding metadata were obtained from the GISAID EpiCoV database (date of accession: 17 June 2020). We applied further quality filters, including selecting only isolates obtained from human hosts (excluding environmental samples and animal hosts), those sequenced for the full length of the genome (sequence size of $29 \mathrm{~kb}$ or greater), and those with high coverage for the reference genome $(<1 \% \mathrm{~N}$ content, $<0.05 \%$ unique mutations, no unverified indel mutations $)$.

To ensure alignment accuracy, all nonstandard unverified nucleotide masking was changed to $\mathrm{N}$ due to the specifications of the alignment software, using the Linux sed command, and the isolates were aligned against the SARS-CoV-2 reference genome (NCBI Reference Sequence 
87 NC_045512.2, available at https://www.ncbi.nlm.nih.gov/nuccore/NC_045512.2) using the

88 MAFFT (v7.450) alignment software (Katoh et al., 2002), using the parameters outlined in the

89 software manual for aligning closely related viral genomes (available at

90 https://mafft.cbrc.jp/alignment/software/closelyrelatedviralgenomes.html). Variant sites in the

91 isolates were annotated using snp-sites (2.5.1), bcftools (1.10.2), and ANNOVAR (release date

9224 October 2019) software (Wang, Li \& Hakonarson, 2010; Page et al., 2016), to identify

93 whether a given mutation was synonymous or nonsynonymous. In addition, the 5' untranslated

94 region of the genome (bases 1-265) and the 100 nucleotides at the 3' end were removed from the

95 alignment and annotation files due to a high number of gaps and unidentified nucleotides. We

96 further removed any sequences with incomplete sequencing location or date data in order to

97 avoid complications in downstream analyses. Following the filters, 29,600 genomes were used

98 for the analyses.

99

100 Mutation density calculation

101 Variants were categorized as synonymous and nonsynonymous following annotation by

102 ANNOVAR, with intergenic or terminal mutations being considered synonymous. Gene

103 mutation densities were calculated separately for synonymous and nonsynonymous mutations, as

104 well as the total of SNVs, for each isolate, using a non-reference nucleotides per kilobase of

105 region metric. Mutation densities were calculated for the combined membrane glycoprotein (M)

106 and envelope glycoprotein (E) genes (MoE), the surface glycoprotein gene (S), and the whole

107 genome. 


\section{Statistical Analysis}

109 Descriptive statistics for continuous variable days were calculated with mean, standard deviation,

110 median, and interquartile range. Kolmogorov-Smirnov test was used to check the normality

111 assumption of the continuous variables. In cases of non-normally distributed data, the Wilcoxon

112 rank-sum (Mann-Whitney U) test was performed to determine whether the difference between

113 the two MoE status groups was statistically significant. The Fisher's exact test and the Pearson

114 chi-square test were used for the analysis of categorical variables. The univariate logistic

115 regression method was utilized to assess the mutations associated with MoE status in single

116 variables, and then multiple logistic regression method was performed. The final multiple

117 logistic regression model was executed with the backward stepwise method. The relationship

118 between mutation density and time in isolates with mutations of interest, as well as in the group

119 comprising all isolates, was examined via non-polynomial linear regression model and

120 Spearman's rank correlation. A p-value of less than 0.05 was considered statistically significant.

121 All statistical analyses were performed using IBM SPSS version 25.0 (Chicago, IL, USA).

\section{Results and Discussion}

123 Increases in the mutation load of SARS-CoV-2 are unevenly distributed across its genome

124 To identify the trends in SARS-CoV-2 mutation load over time, we calculated the average

125 mutation density per day for all isolates for whole genome, $\mathrm{S}$ gene, and MoE regions, capping

126 outliers at the $95^{\text {th }}$ and $5^{\text {th }}$ percentile values to minimize the potential effects of sequencing errors

127 (Fig. 1). Our results show that both at the genome level and the S gene, a very strong positive

128 correlation between average mutation density and time. In comparison, MoE has a weak positive

129 correlation, with a wider spread of mean density in early and late periods compared to the

130 genome and the $\mathrm{S}$ gene. This is consistent with reduced selective pressure on the $\mathrm{M}$ and $\mathrm{E}$ genes, 
131 as has previously been described (Dilucca et al., 2020b). The top nonsynonymous mutation is

132 23403A $>\mathrm{G}$ (in 22271 isolates), responsible for the D614G substitution in the spike protein,

133 followed by the $14408 \mathrm{C}>\mathrm{T}$ mutation (in 22226 isolates) in the nsp12 region of the Orflab gene,

134 causing P323L substitution in the RdRp protein, and the $28144 \mathrm{C}>\mathrm{T}$ mutation (in 3081 isolates),

135 responsible for the L84S substitution in the Orf8 protein. The most common synonymous

136 mutation is the $8782 \mathrm{C}>\mathrm{T}$ mutation (in 3047 isolates), and is found on the nsp 4 coding region of

137 the Orflab gene. For the $\mathrm{S}$ gene, the most frequent synonymous mutation is the $23731 \mathrm{C}>\mathrm{T}$

138 mutation (in 622 isolates), and the second most common nonsynonymous mutation, after the

139 aforementioned D614G mutation, is $25350 \mathrm{C}>\mathrm{T}$ (in 215 isolates), responsible for the P1263L

140 substitution. For MoE, the most common synonymous and nonsynonymous mutations are

$14126735 \mathrm{C}>\mathrm{T}$ (in 341 isolates) and $27046 \mathrm{C}>\mathrm{T}$ (in 530 isolates), respectively, both of which are

142 found in the $\mathrm{M}$ gene, and the latter of which causes T175M amino acid substitution. Other than

143 the D614G mutation, all of the mentioned mutations are $\mathrm{C}>\mathrm{T}$ substitutions, the prevalence of

144 which in T- or A-rich regions of the SARS-CoV-2 genome have been previously documented

145 (Simmonds, 2020).

\section{Mutations in RNA synthesis complex proteins are associated with higher mutation load}

147 After identifying the increase in mutation load over time, which was more prominent in genes

148 with high functional impact (S, Orflab) compared to other structural genes (M, E, N), as seen in

149 Figure 1 and Supplementary Figures 1 and 2, we sought to examine possible associations of

150 variants in proteins involved in SARS-CoV-2 genome replication with the increase. We first

151 identified the five most frequently observed mutations for nsps 7, 8, 12 (also known as RdRp)

152 and 14, four of the proteins cleaved from the Orflab polyprotein and are involved in the RNA

153 polymerization, followed by analyzing the association of each mutation with the presence of 
154 MoE mutations (hereafter referred to as MoE status) using the chi-square test. 12 out of the 20

155 mutations were found to have a significant association with MoE status (p-value $<0.05)$ (Table

156 1). Compared to our previous findings on the top 10 nsp12 mutations (Eskier et al. 2020), which

157 was based on an analysis of 11,208 samples as of 5 May 2020, 13536C $>\mathrm{T}$ and $13862 \mathrm{C}>\mathrm{T}$ have

158 increased in rank of appearance, from $6^{\text {th }}$ and $7^{\text {th }}$ to $4^{\text {th }}$ and $5^{\text {th }}$, respectively, and decreased in $\mathrm{p}$ -

159 value to show statistically significant associations. In addition, the $13730 \mathrm{C}>\mathrm{T}$ mutation have

160 increased in rank of appearance from $4^{\text {th }}$ to $3^{\text {rd }}$. Out of the other nsps tested, nsp 14 was found to

161 have four significant mutations, while nsp7 had two and nsp8 had one.

\section{Effects of geographical location on MoE status}

163 In addition to time and genotype, we also examined the potential association between the

164 location of isolates and MoE status as a possible confounding factor. We first examined whether

165 there is a significant association between location, defined here as continent the isolate was

166 originally obtained, and MoE status. Our results indicate that there is a strong association

167 between location and MoE status, with the highest percentage of MoE present isolates in Asia

$168(14.5 \%)$, and the percentage ratio in South America (6.5\%) (p-value $<0.001)$. In comparison to

169 our previous findings, South America had a dramatic decrease in MoE present isolate

170 percentage, likely as a result of the increased sequencing efforts (from 118 isolates to 416)

171 removing potential sampling biases or localized founder effects. Africa, Asia, and North

172 America had an increase in MoE present proportion, while Europe, Oceania, and South America

173 showed lowered percentages (Table 2).

174 After observing the potential confounding effect of location on MoE status, we sought to

175 understand whether a location is more or less likely to predict MoE status, using a logistic

176 regression model (Table 3). Comparing each individual region (1) to the other five (0), we found 
177 that Asia, Europe, and North and South America are all possible predictors of MoE status (p-

178 value $<0.05$ ), with Asia and Europe 1.697 and 1.184 times as likely to be MoE present as the

179 other regions, and North and South America 0.589 and 0.650 times as likely, respectively.

180 Using these findings, we created different logistic regression models to identify which of the 12

181 mutations are likely to be independent predictors of MoE status (Table 4). In the single variable

182 model, all 12 mutations we previously identified and location were found to be potential

183 predictors ( $\mathrm{p}$-value $<0.05$ ). Forming final models including the 12 mutations (Final Model A)

184 and the mutations as well as locations (Final Model B), we observed that the predictor effect of

185 two of the mutations nsp $812478 \mathrm{G}>\mathrm{A}$ and nsp $1418998 \mathrm{C}>\mathrm{T}$ do not appear to be sufficiently

186 independent of the other mutations in Final Model A. After adding the location variable to the

187 Final Model A, location remains a significant predictor, with all five non-reference locations less

188 likely to predict MoE than Asia, the reference location, and nsp12 $14805 \mathrm{C}>\mathrm{T}$ is found to not

189 have a predictor effect independent of location $(\mathrm{p}$-value $=0.073)$. Following Final Model B, nine

190 mutations appear to have a significant association with MoE status, independent of other

191 variables: $11916 \mathrm{C}>\mathrm{T}, 12073 \mathrm{C}>\mathrm{T}, 13536 \mathrm{C}>\mathrm{T}, 13730 \mathrm{C}>\mathrm{T}, 13862 \mathrm{C}>\mathrm{T}, 14408 \mathrm{C}>\mathrm{T}, 18060 \mathrm{C}>\mathrm{T}$,

$19218736 \mathrm{~T}>\mathrm{C}$, and $18877 \mathrm{C}>\mathrm{T}$ ( $\mathrm{p}$-value $<0.05)$.

193 Nsp14 mutations have significant impact on increased genomic mutation density

194 We then examined the effects of each mutation on genomic mutation density to see whether the

195 relationship between the mutations and MoE status are indicative of a genome-wide trend. Due

196 to selection potentially effecting nonsynonymous mutations differentially, we separated the

197 mutations in the two categories and calculated mutation density separately for each category. Our

198 results show that nsp14 mutations show the most consistent association with mutations between

$199 \mathrm{MoE}$ and the whole genome. All three nsp14 mutations $(18060 \mathrm{C}>\mathrm{T}, 18736 \mathrm{~T}>\mathrm{C}$, and $18877 \mathrm{C}>\mathrm{T})$ 
200 which have a significant association with MoE status also show a similar relationship with 201 genomic mutation density (Fig. 2). 18060C $>$ T (L7L) has the lowest odds ratio for MoE status

202 (Table 4), and while it shows a slower increase in synonymous mutation density compared to 203 wildtype isolates (Fig. 2A), it has a significant impact on faster mutation density increase in 204 nonsynonymous mutations (Fig. 2B). In comparison, 18877C >T (L270L) (Fig. 2C-D) and 205 18736T $>$ C (F233L) (Fig. 2E-F) both show a high prediction capacity for MoE and an increased 206 mutation density. In comparison, mutations in nsp7 (Supp. Figs. 3-4) and nsp12 (Supp. Figs. 5-8)

207 show much lower impact on altered mutation density increase rate. $12073 \mathrm{C}>\mathrm{T}$, an nsp7 mutation, 208 displays high divergence from wildtype isolate patterns; however, its low sample size $(\mathrm{n}=16)$ 209 creates a skewed distribution of isolates across time, complicating any potential inference.

\section{Conclusions}

213 Our previous work identified RdRp mutations as contributors to the evolution of the SARS-CoV-

2142 genome and this study confirmed those findings. Furthermore, we hypothesized that mutations

215 of the other critical components of the viral replication and transcription machinery may have

216 similar effects. Our results implicate nsp14 as a source of increased mutation rate in SARS-CoV-

2172 genomes. Three of the five most common nsp 14 mutations, namely $18060 \mathrm{C}>\mathrm{T}, 18736 \mathrm{~T}>\mathrm{C}$ and

$21818877 \mathrm{C}>\mathrm{T}$ are associated with increases in both genome-wide mutational load, as well as MoE

219 status, an alternative indicator of mutational rate and virus evolution. Interestingly all three are

220 located within the ExoN domain, which is responsible for the proofreading activity of nsp14;

221 however, only $18736 \mathrm{~T}>\mathrm{C}$ mutation is non-synonymous (F233L), while $18060 \mathrm{C}>\mathrm{T}$ and 
$22218877 \mathrm{C}>\mathrm{T}$ are synonymous mutations and therefore, only after functional studies it will be

223 possible to understand their effects on viral replication processes.

224 The origins and fates of the three nsp14 mutations are also quite different: Being present in the

225 first case detected in the Washington state of the US in mid-January, 18060C $>$ T mutation has

226 been almost completely confined to the US, as 1,657 of 2,007 isolates ( $82.6 \%$ ) originating from

227 the US (https://bigd.big.ac.cn/ncov/variation/annotation/variant/18060, accessed 6 September

228 2020). On the other hand, $18877 \mathrm{C}>\mathrm{T}$ mutation arising around at the end of January likely in

229 Saudi Arabia and being detected in much less cases $(\mathrm{n}=893)$, is still present in many isolates,

230 most frequently in Saudi Arabia (54.1\%) and Turkey (37.4\%). 18736T $>$ C mutation was first

231 detected in the US at the beginning of March and like the $18060 \mathrm{C}>\mathrm{T}$ mutation, has almost

232 completely been limited to the US (281/362 or $77.6 \%)$. Unlike the other two, this mutation has

233 been detected in only two isolates since 27 May, and not after 1 July 2020. However, it should be

234 noted that $18877 \mathrm{C}>\mathrm{T}$ mutation arose within the dominant $23403 \mathrm{~A}>\mathrm{G} / 14408 \mathrm{C}>\mathrm{T}$ lineage, while

235 the other two nsp14 mutations are in different lineages. Therefore, dominance or disappearance

236 of different nsp14 mutations may have less to do with these particular mutations and more with

237 the co-mutations. Yet, we cannot rule out possible effects of these nsp14 mutations on the fitness 238 of SARS-CoV-2.

239 Previous studies on alphacoronavirus nsp 14 protein had shown that nsp 14, via its exonuclease

240 activity, can modulate host-virus interactions, degrading double-stranded RNA produced during

241 genome replication to suppress immune response, thus increasing viral viability (Becares et al.,

242 2016). SARS-CoV-2 nsp14, due to similar exonuclease activity, is therefore a potential

243 modulator of host interactions, independent of its link to increased mutation load. However, the

244 exact effect of the mutations we identified, two of which are synonymous and may only 
245 indirectly affect protein structure, have to be studied experimentally to show any possible 246 changes in viral property that they might affect. Of note, a recent study where codon usage of

247 SARS-CoV-2 was analyzed in terms of temporal evolution of the virus genome revealed that

248 nsp14 is one of three genes (together with $\mathrm{S}$ and $\mathrm{N}$ genes) that display the highest Codon

249 Adaptation Index (CAI) values (Dilucca et al., 2020a). CAI is a measure of optimal codon usage

250 and indicates how well codons adapt to the host. Based on higher CAI values in nsp14, one could

251 speculate that such mutations have been accumulating preferentially to reach the optimal

252 mutation rate that allows the most advantageous mutation-selection equilibrium for SARS-CoV-

253 2. Indeed, our previous results (Eskier et al., 2020b) indicated that the mutation densities of

254 SARS-CoV-2 genomes are closely related to the pandemic stage and population dynamics

255 directly affects the average mutational load of the viral genome. During the rapid growth stages,

256 such as those observed in March in the UK and the US, replication fidelity can be traded off to

257 gain higher replication rates and broader mutational diversity. However, mutations in the

258 replication machinery that result in too high mutation rates would likely be detrimental and

259 eliminated. On the other hand, a small percentage of the resulting mutations could possibly be

260 advantageous, including those that could confer resistance to antiviral drugs. So far, we or others

261 have not been able to detect such mutations advantageous for the virus, however, higher

262 mutation rates make appearance of such a mutation more likely.

263 We believe that the mutations discussed in this study can be of help to future studies, in both

264 fighting the COVID-19 pandemic, and better understanding of how mutations in coronavirus

265 replication proteins can affect viral viability and replication fidelity in hosts. Also, it is yet to be

266 determined whether COVID-19 cases infected with SARS-CoV-2 that has mutation(s) that are 
267 associated with higher mutation rate respond better to nucleoside analogs, such as remdesivir or

268 ribavirin.

269

270 Additional Information and Declarations

271 Acknowledgement

272 The authors would like to thank Mr. Alirıza Arıbaş from Izmir Biomedicine and Genome Center

273 for his technical assistance. The authors also would like to extend their thanks to Izmir

274 Biomedicine and Genome Center (IBG) COVID19 platform IBG-COVID19 for their support in

275 implementing the study and the Scientific and Technological Research Council of Turkey

276 (TUBITAK) for their financial support of IBG-COVID19.

277 Funding

278 Yavuz Oktay is supported by the Turkish Academy of Sciences Young Investigator Program

279 (TÜBA- GEBİ). The funders had no role in study design, data collection and analysis, decision

280 to publish, or preparation of the manuscript.

281 Grant Disclosures

282 The following grant information was disclosed by the authors:

283 Turkish Academy of Sciences Young Investigator Program (TÜBA- GEBİP).

284 Competing Interests

285 Aslı Suner and Gökhan Karakülah are Academic Editors at PeerJ.

286 Author Contributions

Peer) reviewing PDF | (2020:08:51800:2:0:NEW 21 Sep 2020) 
287 Doğa Eskier, Aslı Suner, Gökhan Karakülah and Yavuz Oktay conceived and designed the 288 experiments, performed the experiments, analyzed the data, prepared figures and/or tables, 289 authored or reviewed drafts of the paper, and approved the final draft.

Data Availability

291

292

293

294

295

296

297

298

299

300

301

302

303

304

305

306

307

The data is available at Mendeley: Eskier, Doğa; Suner, Asli; Oktay, Yavuz; Karakülah, Gökhan (2020), "SARS-CoV-2 GISAID isolates (2020-06-17) genotyping VCF”, Mendeley Data, v1. http://dx.doi.org/10.17632/63t5c7xb4c.1

\section{Supplemental Information}

Supplemental materials are included with this research.

\section{References}

Becares M, Pascual-Iglesias A, Nogales A, Sola I, Enjuanes L, Zuñiga S. 2016. Mutagenesis of Coronavirus nsp14 Reveals Its Potential Role in Modulation of the Innate Immune Response. Journal of virology 90:5399-5414. DOI: 10.1128/JVI.03259-15.

Chan JF-W, Yuan S, Kok K-H, To KK-W, Chu H, Yang J, Xing F, Liu J, Yip CC-Y, Poon RWS, Tsoi H-W, Lo SK-F, Chan K-H, Poon VK-M, Chan W-M, Ip JD, Cai J-P, Cheng VCC, Chen H, Hui CK-M, Yuen K-Y. 2020. A familial cluster of pneumonia associated with the 2019 novel coronavirus indicating person-to-person transmission: a study of a family cluster. Lancet (London, England) 395:514-523. DOI: 10.1016/S0140-6736(20)30154-9.

Dilucca M, Forcelloni S, Georgakilas AG, Giansanti A, Pavlopoulou A. 2020a. Temporal evolution and adaptation of SARS-COV 2 codon usage. bioRxiv:2020.05.29.123976. DOI: $10.1101 / 2020.05 .29 .123976$. 
308 Dilucca M, Forcelloni S, Georgakilas AG, Giansanti A, Pavlopoulou A. 2020b. Codon Usage 309 and Phenotypic Divergences of SARS-CoV-2 Genes. Viruses 12:498. DOI: $10.3390 / \mathrm{v} 12050498$

311 Eckerle LD, Becker MM, Halpin RA, Li K, Venter E, Lu X, Scherbakova S, Graham RL, Baric RS, Stockwell TB, Spiro DJ, Denison MR. 2010. Infidelity of SARS-CoV Nsp14exonuclease mutant virus replication is revealed by complete genome sequencing. PLoS pathogens 6:e1000896. DOI: 10.1371/journal.ppat.1000896.

315 Elbe S, Buckland-Merrett G. 2017. Data, disease and diplomacy: GISAID’s innovative contribution to global health. Global Challenges 1:33-46. DOI: 10.1002/gch2.1018.

Eskier D, Karakülah G, Suner A, Oktay Y. 2020a. RdRp mutations are associated with SARSCoV-2 genome evolution. PeerJ 8:e9587. DOI: 10.7717/peerj.9587.

Eskier D, Suner A, Karakülah G, Oktay Y. 2020b. Mutation density changes in SARS-CoV-2 are related to the pandemic stage but to a lesser extent in the dominant strain with mutations in spike and RdRp. PeerJ 8:e9703. DOI: 10.7717/peerj.9703.

Hadfield J, Megill C, Bell SM, Huddleston J, Potter B, Callender C, Sagulenko P, Bedford T, 323 Neher RA. 2018. Nextstrain: real-time tracking of pathogen evolution. Bioinformatics 34:4121-4123. DOI: 10.1093/bioinformatics/bty407.

Katoh K, Misawa K, Kuma K, Miyata T. 2002. MAFFT: a novel method for rapid multiple sequence alignment based on fast Fourier transform. Nucleic Acids Research 30:30593066. DOI: $10.1093 / \mathrm{nar} / \mathrm{gkf436.}$

Kirchdoerfer RN, Ward AB. 2019. Structure of the SARS-CoV nsp12 polymerase bound to nsp7 and nsp8 co-factors. Nature Communications 10:2342. DOI: 10.1038/s41467-019-102803. 
331 Kochi AN, Tagliari AP, Forleo GB, Fassini GM, Tondo C. 2020. Cardiac and arrhythmic 332 complications in patients with COVID-19. Journal of Cardiovascular Electrophysiology 31:1003-1008. DOI: 10.1111/jce.14479.

334 Lee I-C, Huo T-I, Huang Y-H. 2020. Gastrointestinal and liver manifestations in patients with 335 COVID-19. Journal of the Chinese Medical Association: JCMA 83:521-523. DOI: 10.1097/JCMA.0000000000000319.

Li Y-C, Bai W-Z, Hashikawa T. 2020. The neuroinvasive potential of SARS-CoV2 may play a role in the respiratory failure of COVID-19 patients. Journal of Medical Virology 92:552-555. DOI: 10.1002/jmv.25728.

Ma Y, Wu L, Shaw N, Gao Y, Wang J, Sun Y, Lou Z, Yan L, Zhang R, Rao Z. 2015. Structural basis and functional analysis of the SARS coronavirus nsp14-nsp10 complex. Proceedings of the National Academy of Sciences of the United States of America 112:9436-9441. DOI: 10.1073/pnas.1508686112.

Ogando NS, Ferron F, Decroly E, Canard B, Posthuma CC, Snijder EJ. 2019. The Curious Case 345 of the Nidovirus Exoribonuclease: Its Role in RNA Synthesis and Replication Fidelity. Frontiers in Microbiology 10. DOI: 10.3389/fmicb.2019.01813.

Page AJ, Taylor B, Delaney AJ, Soares J, Seemann T, Keane JA, Harris SR. 2016. SNP-sites: rapid efficient extraction of SNPs from multi-FASTA alignments. Microbial genomics 2:e00056-e000056. DOI: 10.1099/mgen.0.000056.

Peng Q, Peng R, Yuan B, Zhao J, Wang M, Wang X, Wang Q, Sun Y, Fan Z, Qi J, Gao GF, Shi Y. 2020. Structural and Biochemical Characterization of the nsp12-nsp7-nsp8 Core Polymerase Complex from SARS-CoV-2. Cell Reports 31:107774. DOI: 10.1016/j.celrep.2020.107774. 
354 Riou J, Althaus CL. 2020. Pattern of early human-to-human transmission of Wuhan 2019 novel

355

356

357

358

360

361

362

363

364

365

366

367

368

369

370

371

372

373

374

375

376 coronavirus (2019-nCoV), December 2019 to January 2020. Eurosurveillance 25. DOI: 10.2807/1560-7917.ES.2020.25.4.2000058.

Romano M, Ruggiero A, Squeglia F, Maga G, Berisio R. 2020. A Structural View of SARSCoV-2 RNA Replication Machinery: RNA Synthesis, Proofreading and Final Capping. Cells 9. DOI: 10.3390/cells9051267.

Simmonds P. 2020. Rampant $\mathrm{C} \rightarrow \mathrm{U}$ Hypermutation in the Genomes of SARS-CoV-2 and Other Coronaviruses: Causes and Consequences for Their Short- and Long-Term Evolutionary Trajectories. mSphere 5. DOI: 10.1128/mSphere.00408-20.

Subissi L, Posthuma CC, Collet A, Zevenhoven-Dobbe JC, Gorbalenya AE, Decroly E, Snijder EJ, Canard B, Imbert I. 2014. One severe acute respiratory syndrome coronavirus protein complex integrates processive RNA polymerase and exonuclease activities. Proceedings of the National Academy of Sciences 111:E3900-E3909. DOI: 10.1073/pnas.1323705111.

Wang K, Li M, Hakonarson H. 2010. ANNOVAR: functional annotation of genetic variants from high-throughput sequencing data. Nucleic Acids Research 38:e164-e164. DOI: 10.1093/nar/gkq603.

Wong J, Jamaludin SA, Alikhan MF, Chaw L. 2020. Asymptomatic transmission of SARS-CoV2 and implications for mass gatherings. Influenza and Other Respiratory Viruses. DOI: 10.1111/irv.12767.

Wu Y, Xu X, Chen Z, Duan J, Hashimoto K, Yang L, Liu C, Yang C. 2020. Nervous system involvement after infection with COVID-19 and other coronaviruses. Brain, Behavior, and Immunity 87:18-22. DOI: 10.1016/j.bbi.2020.03.031. 
377 Xu L, Liu J, Lu M, Yang D, Zheng X. 2020. Liver injury during highly pathogenic human

378

379

380

381

382

383

384

385

386

387

388

389

390

391

392

393

394

395

396

397

398

coronavirus infections. Liver International: Official Journal of the International Association for the Study of the Liver 40:998-1004. DOI: 10.1111/liv.14435.

Zhu H, Rhee J-W, Cheng P, Waliany S, Chang A, Witteles RM, Maecker H, Davis MM, Nguyen PK, Wu SM. 2020. Cardiovascular Complications in Patients with COVID-19: Consequences of Viral Toxicities and Host Immune Response. Current Cardiology Reports 22:32. DOI: 10.1007/s11886-020-01292-3.

\section{Figure Legends}

Figure 1. The average mutation density per day for genome, $S$ gene, and $M$ and $E$ genes.

(A) The mutation density vs. time for the whole SARS-CoV-2 genome. (B) The mutation density vs. time for the $\mathrm{S}$ gene. (C) The combined mutation density vs. time for the $\mathrm{M}$ and $\mathrm{E}$ genes.

Values in y-axis represent the average number of SNVs in the corresponding day, normalized by kilobase of region of interest. SNV counts of genomes are normalized by capping at the 25- and 75-percentile values to minimize the effects of potential sequencing or assembly artifacts. Correlation scores are calculated using Spearman rank correlation.

Figure 2. The distribution of synonymous and nonsynonymous mutations in isolates carrying nsp14 mutations compared to wildtype isolates. (A-B) Isolates carrying the synonymous $18060 \mathrm{C}>\mathrm{T}$ mutation $(\mathrm{n}=1585)$. (C-D) Isolates carrying the synonymous $18877 \mathrm{C}>\mathrm{T}$ mutation $(\mathrm{n}=893)$. $(\mathrm{E}-\mathrm{F})$ Isolates carrying the nonsynonymous $18736 \mathrm{~T}>\mathrm{C}$ mutation $(\mathrm{n}=236)$. Wildtype isolates in all graphs carry the reference nucleotide for the nine positions of 
399 interest $(11916,12073,13536,13730,13862,14408,18060,18736,18877)(\mathrm{n}=5910)$.

400 Correlation scores are calculated using Spearman rank correlation. 


\section{Table 1 (on next page)}

Comparisons of MoE and nsp mutations 
Table 1. Comparisons of MoE and nsp mutations.

\begin{tabular}{|c|c|c|c|c|c|c|c|c|c|}
\hline \multirow{2}{*}{ NSP } & \multirow{2}{*}{ Mutations } & \multirow{2}{*}{ Values } & \multicolumn{2}{|c|}{ MoE Absent } & \multicolumn{2}{|c|}{ MoE Present } & \multicolumn{2}{|c|}{ Total } & \multirow[b]{2}{*}{$\mathbf{p}$} \\
\hline & & & $\mathbf{n}$ & $\%$ & $\mathbf{n}$ & $\%$ & $\mathbf{n}$ & $\%$ & \\
\hline \multirow{10}{*}{ nsp7 } & $11916 C>T$ & Absent & 26326 & 98.4 & 2833 & 99.7 & 29159 & 98.5 & \multirow{2}{*}{$<0.001^{*}$} \\
\hline & S3884L & Present & 433 & 1.6 & 8 & 0.3 & 441 & 1.5 & \\
\hline & $12076 \mathrm{C}>\mathrm{T}$ & Absent & 26735 & 99.9 & 2837 & 99.9 & 29572 & 99.9 & \multirow{2}{*}{0.339} \\
\hline & N3937N & Present & 24 & 0.1 & 4 & 0.1 & 28 & 0.1 & \\
\hline & $11919 C>T$ & Absent & 26738 & 99.9 & 2840 & 100.0 & 29578 & 99.9 & \multirow{2}{*}{0.717} \\
\hline & S3885F & Present & 21 & 0.1 & 1 & - & 22 & 0.1 & \\
\hline & $12073 C>T$ & Absent & 26750 & 100.0 & 2834 & 99.8 & 29584 & 99.9 & \multirow{2}{*}{$<0.001^{*}$} \\
\hline & D3936D & Present & 9 & - & 7 & 0.2 & 16 & 0.1 & \\
\hline & $11962 C>T$ & Absent & 26746 & 100.0 & 2840 & 100.0 & 29586 & 100.0 & \multirow{2}{*}{1.000} \\
\hline & L3899L & Present & 13 & - & 1 & - & 14 & - & \\
\hline \multirow{10}{*}{ nsp8 } & $12478 G>A$ & Absent & 26757 & 100.0 & 2750 & 96.8 & 29507 & 99.7 & \multirow{2}{*}{$<0.001^{*}$} \\
\hline & M4071I & Present & 2 & - & 91 & 3.2 & 93 & 0.3 & \\
\hline & $12550 \mathrm{G}>\mathrm{A}$ & Absent & 26697 & 99.8 & 2841 & 100.0 & 29538 & 99.8 & \multirow[b]{2}{*}{ - } \\
\hline & L4095L & Present & 62 & 0.2 & - & - & 62 & 0.2 & \\
\hline & $12415 C>T$ & Absent & 26725 & 99.9 & 2841 & 100.0 & 29566 & 99.9 & \multirow[b]{2}{*}{ - } \\
\hline & $\mathrm{N} 4050 \mathrm{~N}$ & Present & 34 & 0.1 & - & - & 34 & 0.1 & \\
\hline & $12557 A>G$ & Absent & 26729 & 99.9 & 2841 & 100.0 & 29566 & 99.9 & \multirow{2}{*}{ - } \\
\hline & I4098V & Present & 30 & 0.1 & - & - & 30 & 0.1 & \\
\hline & $12400 \mathrm{C}>\mathrm{T}$ & Absent & 26734 & 99.9 & 2840 & 100.0 & 29574 & 99.9 & \multirow{2}{*}{0.508} \\
\hline & L4045L & Present & 25 & 0.1 & 1 & - & 26 & 0.1 & \\
\hline \multirow{10}{*}{ nsp12 } & $14408 C>T$ & Absent & 7498 & 28.0 & 702 & 24.7 & 8200 & 27.7 & \multirow{2}{*}{$<0.001^{*}$} \\
\hline & P4715L & Present & 19261 & 72.0 & 2139 & 75.3 & 21400 & 72.3 & \\
\hline & $14805 \mathrm{C}>\mathrm{T}$ & Absent & 24397 & 91.2 & 2704 & 95.2 & 27101 & 91.6 & \multirow{2}{*}{$<0.001^{*}$} \\
\hline & Y4847Y & Present & 2362 & 8.8 & 137 & 4.8 & 2499 & 8.4 & \\
\hline & $13730 \mathrm{C}>\mathrm{T}$ & Absent & 26238 & 98.1 & 2820 & 99.3 & 29058 & 98.2 & \multirow{2}{*}{$<0.001^{*}$} \\
\hline & A4489V & Present & 521 & 1.9 & 21 & 0.7 & 542 & 1.8 & \\
\hline & $13536 \mathrm{C}>\mathrm{T}$ & Absent & 26469 & 98.9 & 2823 & 99.4 & 29292 & 99.0 & \\
\hline & Y4424Y & Present & 290 & 1.1 & 18 & 0.6 & 308 & 1.0 & $0.025^{4}$ \\
\hline & $13862 C>T$ & Absent & 26535 & 99.2 & 2833 & 99.7 & 29368 & 99.2 & \\
\hline & T4533I & Present & 224 & 0.8 & 8 & 0.3 & 232 & 0.8 & $0.001^{*}$ \\
\hline & $18060 \mathrm{C}>\mathrm{T}$ & Absent & 25247 & 94.3 & 2768 & 97.4 & 28015 & 94.6 & \\
\hline & L5932L & Present & 1512 & 5.7 & 73 & 2.6 & 1585 & 5.4 & $0.001^{*}$ \\
\hline & $18877 C>T$ & Absent & 26185 & 97.9 & 2522 & 88.8 & 28707 & 97.0 & \\
\hline & L6205L & Present & 574 & 2.1 & 319 & 11.2 & 893 & 3.0 & $0.001^{*}$ \\
\hline & $18998 C>T$ & Absent & 26454 & 98.9 & 2836 & 99.8 & 29290 & 99.0 & \\
\hline nsp14 & A6245V & Present & 305 & 1.1 & 5 & 0.2 & 310 & 1.0 & $0.001 *$ \\
\hline & $18736 \mathrm{~T}>\mathrm{C}$ & Absent & 26751 & 100.0 & 2613 & 92.0 & 29364 & 99.2 & $0001 *$ \\
\hline & F6158L & Present & 8 & - & 228 & 8.0 & 236 & 0.8 & 0.001 \\
\hline & $19524 C>T$ & Absent & 26530 & 99.1 & 2825 & 99.4 & 29355 & 99.2 & \\
\hline & L6420L & Present & 229 & 0.9 & 16 & 0.6 & 245 & 0.8 & 0.102 \\
\hline & Tot & & 26759 & 100.0 & 2840 & 100.0 & 29600 & 100.0 & \\
\hline
\end{tabular}


Table 2 (on next page)

Distribution of MoE across geographical locations.

*p-value $<0.05$ was statistically significant. 
Table 2. Distribution of MoE across geographical locations.

\begin{tabular}{|c|c|c|c|c|c|c|c|}
\hline \multirow[t]{2}{*}{ Locations } & \multicolumn{2}{|c|}{ MoE absent } & \multicolumn{2}{|c|}{ MoE present } & \multicolumn{2}{|c|}{ Total } & \multirow[t]{2}{*}{$\mathbf{p}$} \\
\hline & $\mathbf{n}$ & $\%$ & $\mathbf{n}$ & $\%$ & $\mathbf{n}$ & $\%$ & \\
\hline Asia & 2319 & 85.5 & 394 & 14.5 & 2713 & 100.0 & \multirow{7}{*}{$<0.001^{*}$} \\
\hline Africa & 297 & 90.3 & 32 & 9.7 & 329 & 100.0 & \\
\hline South America & 389 & 93.5 & 27 & 6.5 & 416 & 100.0 & \\
\hline Europe & 14879 & 89.8 & 1697 & 10.2 & 16576 & 100.0 & \\
\hline North America & 7401 & 93.4 & 522 & 6.6 & 7923 & 100.0 & \\
\hline Oceania & 1474 & 89.7 & 169 & 10.3 & 1643 & 100.0 & \\
\hline Total & 26759 & 90.4 & 2841 & 9.6 & 29600 & 100.0 & \\
\hline
\end{tabular}




\section{Table 3(on next page)}

Logistic regression model of MoE and location on single variables.

Each location was represented as itself (1) and others (0). OR: Odds-Ratio; C.I.: confidence interval, *p-value $<0.05$ was statistically significant. 
1 Table 3. Logistic regression model of $\mathrm{MoE}$ and location on single variables. Each location was 2 represented as itself (1) and others (0).

3

4
5

\begin{tabular}{c|ccl}
\hline Locations & $\mathbf{p}$ & OR & $\mathbf{9 5 \%}$ C.I. \\
\hline Asia & $<0.001^{*}$ & 1.697 & 1.513 to 1.903 \\
Africa & 0.937 & 1.015 & 0.703 to 1.465 \\
South America & $0.032 *$ & 0.650 & 0.439 to 0.963 \\
Europe & $<0.001 *$ & 1.184 & 1.095 to 1.281 \\
North America & $<0.001 *$ & 0.589 & 0.533 to 0.650 \\
Oceania & 0.330 & 1.085 & 0.921 to 1.278 \\
\hline
\end{tabular}

OR, Odds-Ratio; C.I.: confidence interval, ${ }^{*}$ p-value $<0.05$ was statistically significant. 


\section{Table 4 (on next page)}

Logistic regression model of MoE on single variables and a final model.

(Final Model A) Logistic regression model of ten mutations on final model. (Final Model B) Logistic regression model of four mutations and location on final model. OR: Odds-Ratio; C.I.: confidence interval; Multiple logistic regression final model was executed on all these statistically significant variables, included together in the model, and selected with backward stepwise method; *p-value $<0.05$ was statistically significant. 
Table 4. Logistic regression model of MoE on single variables and a final model. (Final Model A) Logistic regression model of ten

mutations on final model. (Final Model B) Logistic regression model of four mutations and location on final model.

\begin{tabular}{|c|c|c|c|c|c|c|c|c|c|}
\hline \multirow[b]{2}{*}{ Mutations } & \multicolumn{3}{|c|}{ Single Variables } & \multicolumn{3}{|c|}{ Final Model A } & \multicolumn{3}{|c|}{ Final Model B } \\
\hline & $\mathbf{p}$ & OR & 95\% C.I. & $\mathbf{p}$ & OR & 95\% C.I. & $\mathbf{p}$ & OR & 95\% C.I. \\
\hline Nsp7.11916 & $<0.001^{*}$ & 0.172 & 0.085 to 0.346 & $<0.001^{*}$ & 0.180 & 0.089 to 0.363 & $0.001 *$ & 0.314 & 0.154 to 0.641 \\
\hline Nsp7.12076 & 0.403 & 1.571 & 0.545 to 4.530 & - & - & - & - & - & - \\
\hline Nsp7.11919 & 0.433 & 0.448 & 0.060 to 3.334 & - & - & - & - & - & - \\
\hline Nsp7.12073 & $<0.001^{*}$ & 7.341 & 2.732 to 19.728 & $<0.001^{*}$ & 8.108 & 3.009 to 21.847 & $<0.001^{*}$ & 9.164 & 3.311 to 25.361 \\
\hline Nsp7.11962 & 0.756 & 0.724 & 0.095 to 5.540 & - & - & - & - & - & - \\
\hline Nsp8.12478 & $<0.001^{*}$ & 442.707 & 108.996 to 1798.139 & - & - & - & - & - & - \\
\hline Nsp8.12550 & 0.997 & - & - & - & - & - & - & - & - \\
\hline Nsp8.12415 & 0.998 & - & - & - & - & - & - & - & - \\
\hline Nsp8.12557 & 0.998 & - & - & - & - & - & - & - & - \\
\hline Nsp8.12400 & 0.388 & 0.377 & 0.051 to 2.780 & - & - & - & - & - & - \\
\hline Nsp12.14408 & $<0.001 *$ & 1.186 & 1.085 to 1.297 & $<0.001^{*}$ & 1.310 & 1.144 to 1.500 & $<0.001 *$ & 1.662 & 1.435 to 1.926 \\
\hline Nsp12.14805 & $<0.001^{*}$ & 0.523 & 0.439 to 0.625 & $0.007 *$ & 0.746 & 0.603 to 0.923 & 0.073 & 0.817 & 0.655 to 1.019 \\
\hline Nsp12.13730 & $<0.001^{*}$ & 0.375 & 0.242 to 0.581 & $0.002 *$ & 0.497 & 0.317 to 0.778 & $<0.001^{*}$ & 0.393 & 0.250 to 0.619 \\
\hline Nsp12.13536 & $0.026^{*}$ & 0.582 & 0.361 to 0.938 & $0.044^{*}$ & 0.611 & 0.379 to 0.987 & $0.009 *$ & 0.528 & 0.327 to 0.855 \\
\hline Nsp12.13862 & $0.002 *$ & 0.335 & 0.165 to 0.678 & $0.004^{*}$ & 0.355 & 0.175 to 0.720 & $0.001 *$ & 0.293 & 0.144 to 0.594 \\
\hline Nsp14.18060 & $<0.001^{*}$ & 0.440 & 0.347 to 0.559 & $0.001 *$ & 0.625 & 0.479 to 0.816 & $0.001 *$ & 1.658 & 1.244 to 2.209 \\
\hline Nsp14.18877 & $<0.001 *$ & 5.770 & 5.002 to 6.656 & $<0.001^{*}$ & 5.543 & 4.793 to 6.409 & $<0.001 *$ & 6.437 & 5.483 to 7.557 \\
\hline Nsp14.18998 & $<0.001^{*}$ & 0.153 & 0.063 to 0.370 & - & - & - & - & - & - \\
\hline Nsp14.18736 & $<0.001^{*}$ & 291.773 & 144.002 to 591.182 & $<0.001^{*}$ & 368.884 & 180.195 to 755.153 & $<0.001^{*}$ & 970.884 & 469.324 to 2008.453 \\
\hline Nsp14.19524 & 0.104 & 0.656 & 0.395 to 1.091 & - & - & - & - & - & - \\
\hline Location & $<0.001^{*}$ & - & - & & & & $<0.001^{*}$ & & \\
\hline Africa & $0.019 *$ & 0.634 & $0.434-0.927$ & - & - & - & $0.017 *$ & 0.580 & $0.391-0.860$ \\
\hline South America & $<0.001^{*}$ & 0.409 & $0.273-0.612$ & - & - & - & $<0.001^{*}$ & 0.302 & $0.198-0.461$ \\
\hline Europe & $<0.001^{*}$ & 0.671 & $0.597-0.755$ & - & - & - & $<0.001^{*}$ & 0.681 & $0.591-0.785$ \\
\hline North America & $<0.001^{*}$ & 0.415 & $0.361-0.477$ & - & - & - & $<0.001^{*}$ & 0.228 & $0.192-0.271$ \\
\hline Oceania & $<0.001^{*}$ & 0.675 & $0.557-0.817$ & - & - & - & $<0.001 *$ & 0.536 & $0.428-0.670$ \\
\hline
\end{tabular}


3 OR, Odds-Ratio; C.I.: confidence interval; Multiple logistic regression final model was executed on all these statistically significant variables, included together in the model, and

selected with backward stepwise method; *p-value $<0.05$ was statistically significant. 


\section{Figure 1}

The average mutation density per day for genome, $\mathrm{S}$ gene, and $\mathrm{M}$ and $\mathrm{E}$ genes.

(A) The mutation density vs. time for the whole SARS-CoV-2 genome. (B) The mutation

density vs. time for the $\mathrm{S}$ gene. (C) The combined mutation density vs. time for the $\mathrm{M}$ and $\mathrm{E}$ genes. Values in $y$-axis represent the average number of SNVs in the corresponding day, normalized by kilobase of region of interest. SNV counts of genomes are normalized by capping at the 25- and 75-percentile values to minimize the effects of potential sequencing or assembly artifacts. Correlation scores are calculated using Spearman rank correlation. 


\section{Average Density vs Days - All}

A.

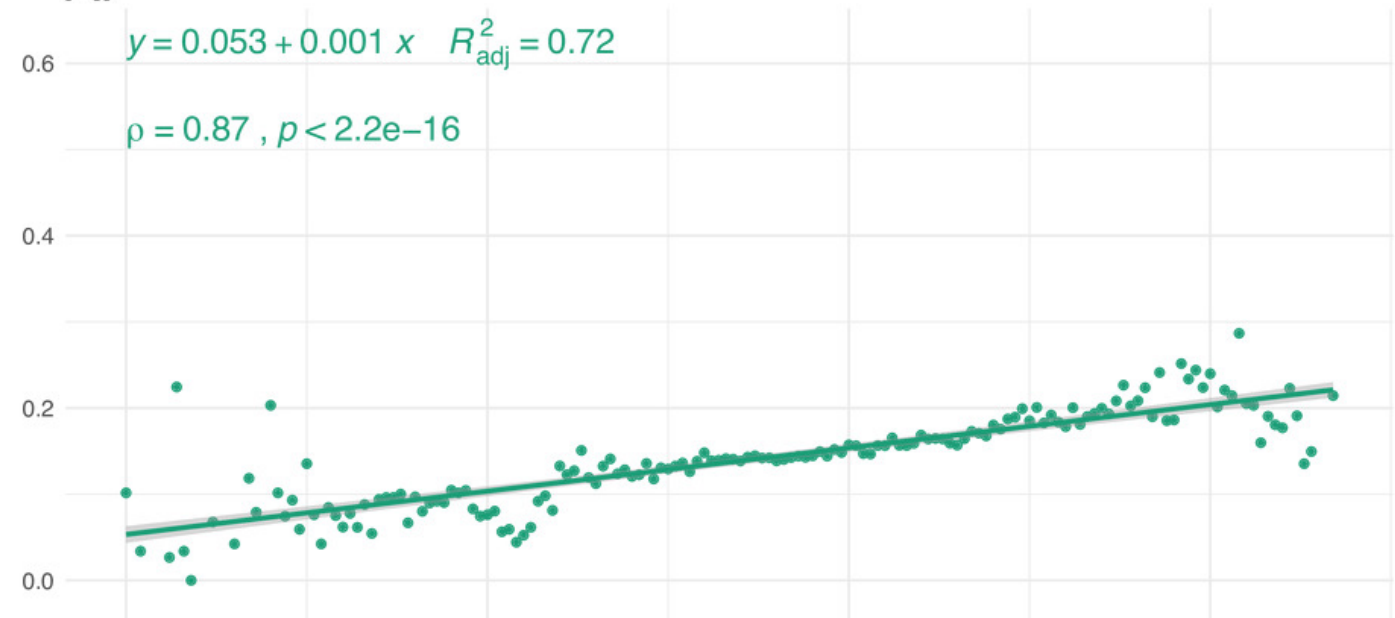

Region

te Genome

at $S$

ar MoE

B.

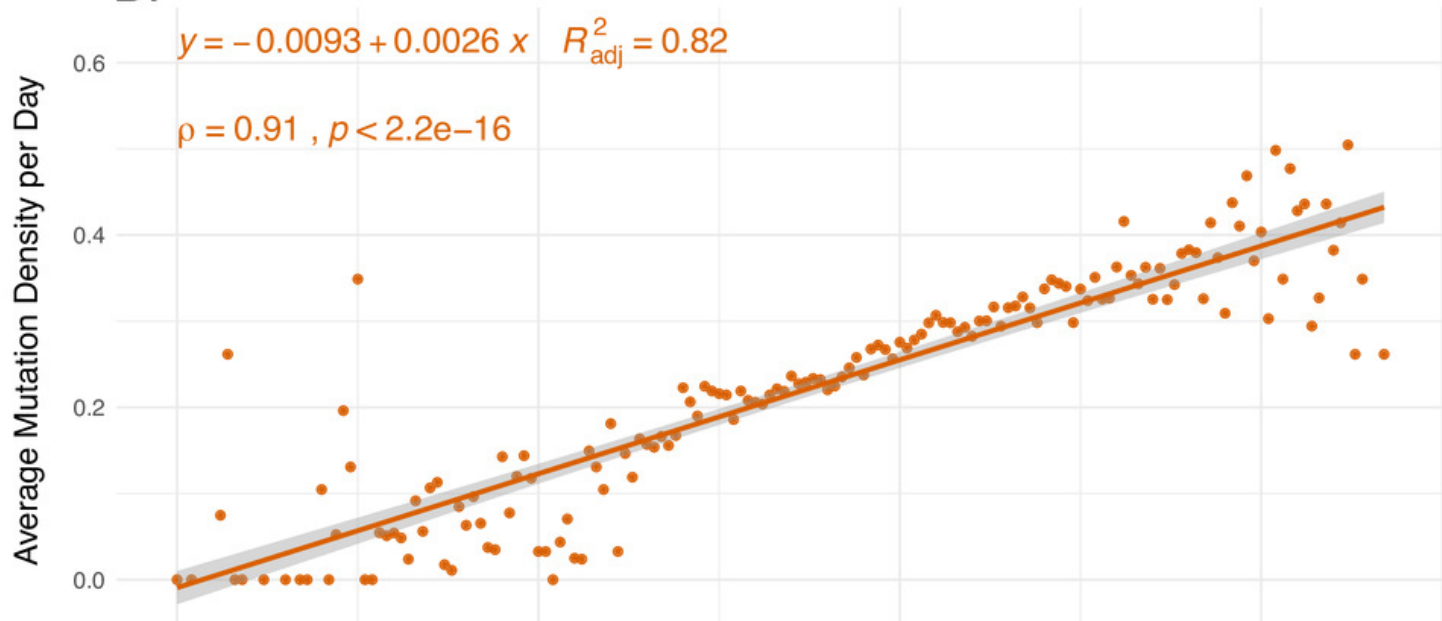

C.

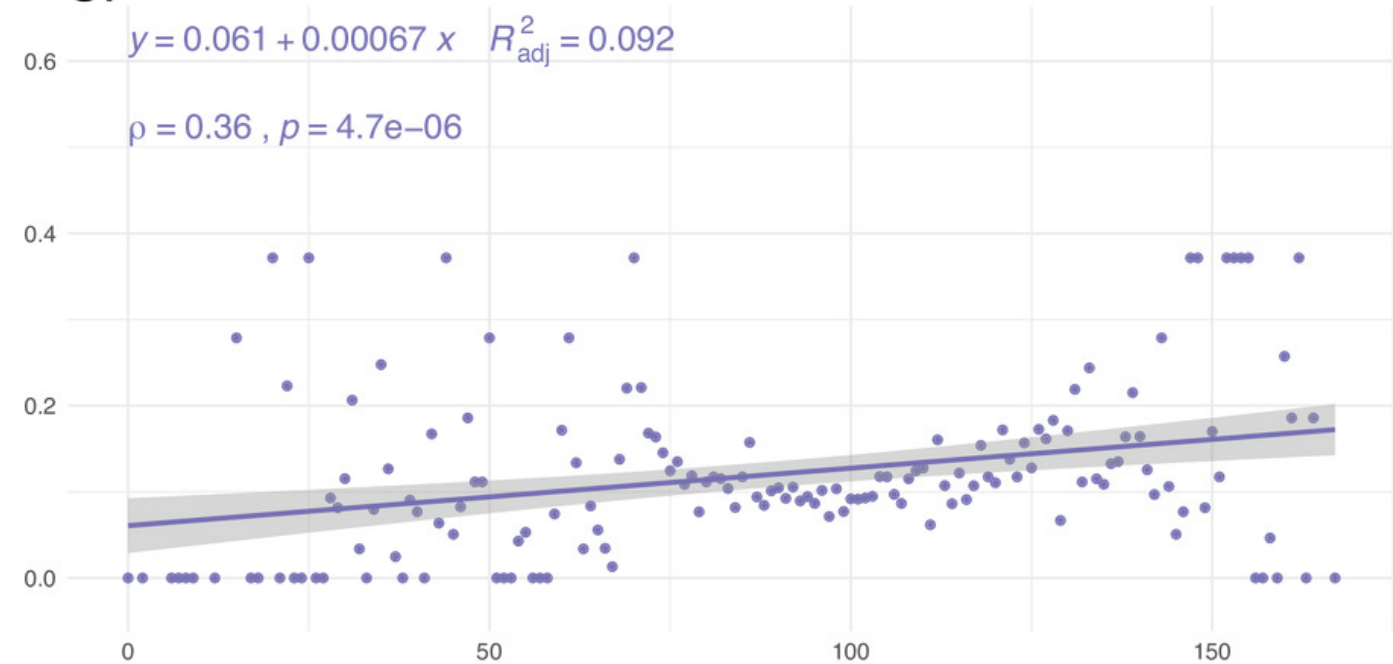

Days 


\section{Figure 2}

The distribution of synonymous and nonsynonymous mutations in isolates carrying nsp14 mutations compared to wildtype isolates.

(A-B) Isolates carrying the synonymous $18060 C>T$ mutation $(n=1585)$. (C-D) Isolates carrying the synonymous $18877 \mathrm{C}>$ T mutation $(n=893)$. (E-F) Isolates carrying the nonsynonymous $18736 \mathrm{~T}>\mathrm{C}$ mutation $(n=236)$. Wildtype isolates in all graphs carry the reference nucleotide for the nine positions of interest (11916, 12073, 13536, 13730, 13862, $14408,18060,18736,18877)(n=5910)$. Correlation scores are calculated using Spearman rank correlation. 


\section{Synonymous}

A.

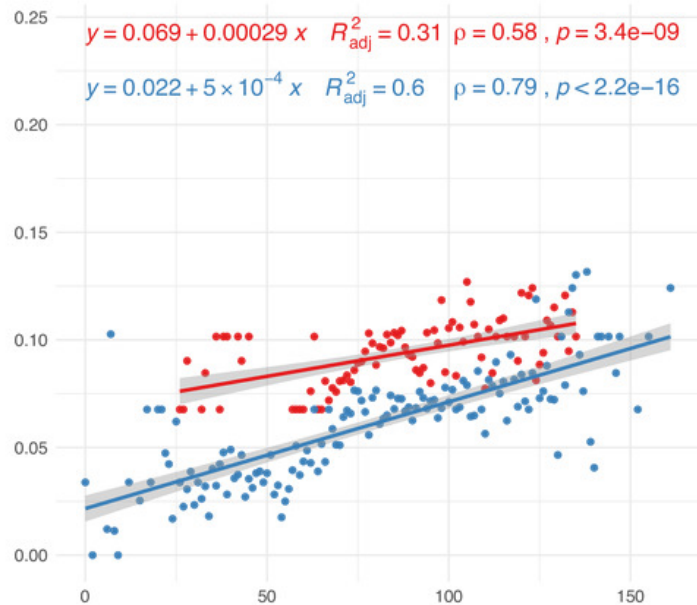

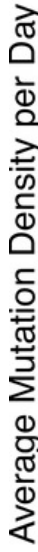

C.

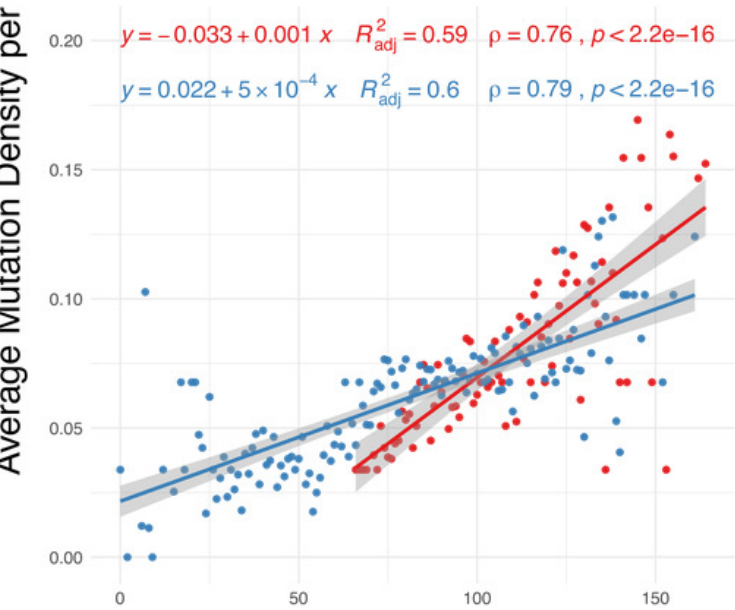

E.

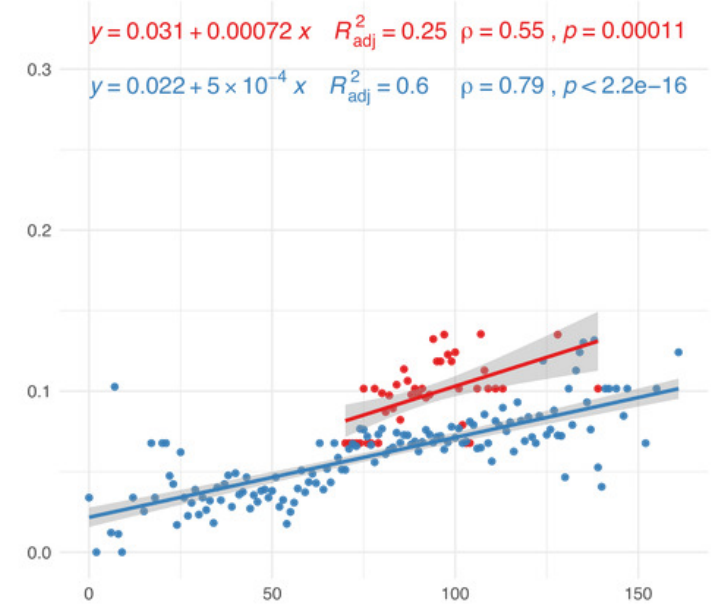

B.

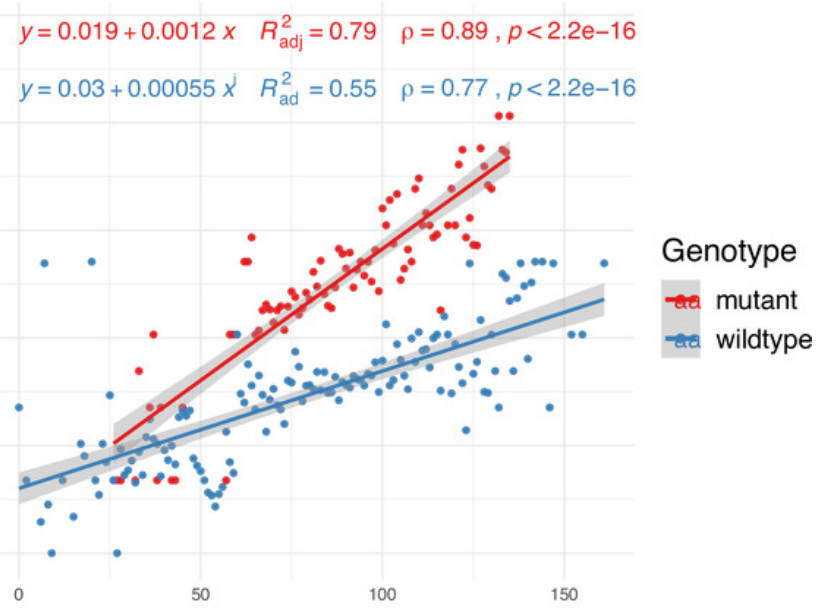

D.

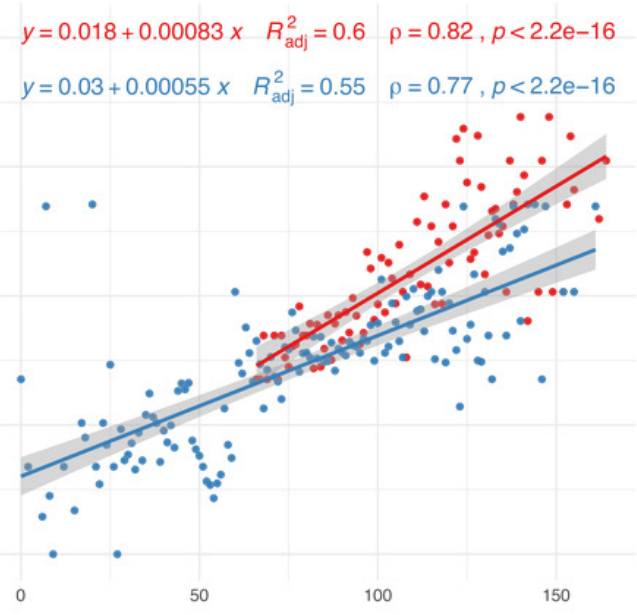

F.

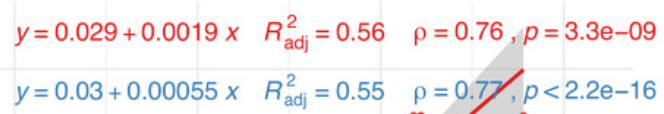

$y=0.03+0.00055 x \quad R_{\text {adj }}^{2}=0.55 \quad \rho=0.77 /, p<2.2 e-16$

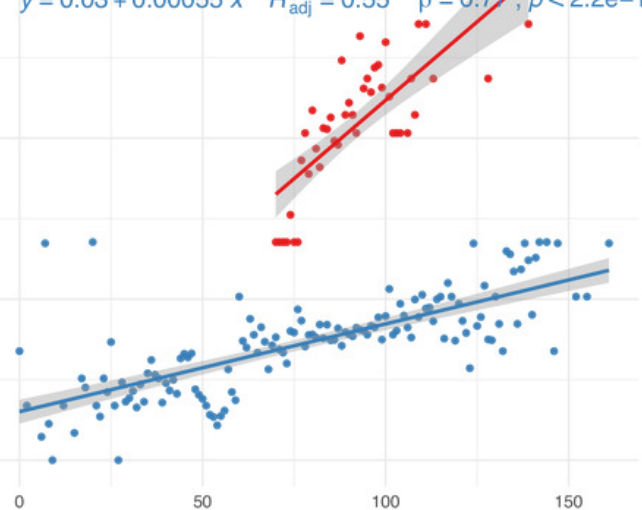

Days 